\title{
Software Defined Networking Based Optical Network: A review
}

\author{
${ }^{1}$ Firas Mahmood Mustafa \\ ${ }^{1}$ Department of Communication, Nawroz University and (Duhok Polytechnic University), Duhok, Kurdistan Region \\ of Iraq
}

\begin{abstract}
In the field of networking, software-defined networking (SDN) has obtained a lot of concentration from both academic and industry, and it aims to provide a flexible and programmable level of control, beside obtain efficient control and management of network systems. For such reasons, the software-defined networks (SDN) can be deemed as an essential task to accomplish these requirements. In the datacenters and networks, the SDN is used to allow the administrators of the networks to start programming, controlling, changing, and managing dynamically the network behavior with open interfaces and a reflection of lower-level functionality because the need for SDN-like switching technology has become evident for many users of network equipment, especially in large data centers. There are many algorithms and applications that have been considered in SDN such as (FP-MA), EON, (EQUAL-APP) (VONCR$\mathrm{APP}$ ), and (T-SDN) as use cases for approval purposes because the SDN provides several focal points to the power, operation, and administration of extensive range networks. This paper aims to review Optical Network using SDN, where many types of research papers are present techniques to improve near-optimal traffic engineering and management; measurement and monitoring of the significant parameters of the optical networks and manage the cross-layer issues such as debugging and testing.
\end{abstract}

KEY WORDS: Control and Management in Network; Optical Network; Software Defined Networking; SDN for Optical Networks; Network traffic engineering.

\section{Introduction}

The world nowadays is an associated entity, and the age is the era of the cloud computing. Suppliers are required to appropriate resources around the globe to give every minute of every day uptime in the event a storage facility goes down. Applications requiring quick reallocation of resources have changed the requests set on transport systems, making SDN an alluring other option to current administration technology. Modern optical network systems are colossal in size and scope. The enormous measure of information is taken care of by these systems, which makes numerous challenges for the administrators and the sellers for arranging management and control. An incorporated system for the general arrangement identified with the operation, control, and administration is required for optical networks.

Software Defined Networking (SDN) is such a system (framework) which makes the control and administration capacities adaptable and cost-effective. It is an unfurling organizing viewpoint that can eliminate the natural restrictions of continuous infrastructural operations. It keeps away from vertical coordination by isolating the control plane from the data plane and the routing and switching techniques to course the movement (traffic). An imperative result of the SDN structure is the softening of system strategies up data sending. The data plane does whatever control plane and the management plane request from it through the SDN programming. Optical systems are developing with emerging advanced technologies. SDN gives adaptability and readiness at each level of the network. 
Optical networks require SDN to break free of these constraints and empower dynamic provisioning, advance network use, and production of new sources of income. Transporter networks incorporate different network designs and equipment types, SDN control plane ready used to help multi-domain, multi-layer resource allocation, and optimization. Even though SDN is a new field of research, several extreme and careful investigations have been done on it. SDN has recently emerged as a significant network architecture empowering the immediate programmability of sending capacities and the powerful abstraction of the hidden foundation (Kreutz et al., 2015). Therefore, the modern computer network has developed, SDN represents another way to deal with computer networking that attempts to address the weaknesses of the current worldview. SDN is an on a very basic level novel approach to programming the switches used in modern data networks having a very highly scalable and centralized network control architecture. Enthusiasm for SDN goes a long way past research and engineering communities intrigued by this new Internet switching technology. An optical network is a communications network in which transmission links are made up of optical fibers, and its engineering is intended to abuse the optical fiber favorable circumstances (Mustafa, 2017). The most common utilization of SDN is to control the network in the electric layers, including OSI/ISO layers 2 and above. However, SDN can be used much further. Professionals and scientists noticed that combining management and control of many layers at the same time can provide benefits (Kantor et al., 2019).

The main objective of this paper is to survey the literature on SDN focusing on optical and electrical layers. The goal is to describe the significant developments in the region of communication using SDN in the optical network, provide a deep and comprehensive understanding of this paradigm, its related technologies, and its domains of application, as well as major issues that need to be solved towards sustaining its success.

\section{OPTICAL NETWORKS}

Optical networking is a method for communication that utilizations signals encoded onto light to transmit data among different nodes of a broadcast communications network. Operate from the limited range of a local-area network (LAN) or over a wide-area network (WAN), which can cross metropolitan and territorial regions the distance to national, universal and transoceanic distances. It is a type of optical communication that depends on optical amplifiers, lasers or LEDs and wave division multiplexing (WDM) to transmit huge amounts of information, for the most part crosswise over fiberoptic cables. Since it is equipped for accomplishing to a great degree high transmission capacity, it is enabling technology for today's Internet and the communication networks that transmit most by far of all human and machine-to-machine data. Components of an optical networking system include Fiber. Multi-mode or singlemode, Laser or LED light source, Multiplexer/demultiplexer, likewise called mux/demux, filter, or crystal. These can incorporate Optical Add/Drop Multiplexer (OADM) and Reconfigurable Optical Add/Drop Multiplexer (ROADM), Optical change, to coordinate light between ports without an optical-electrical-optical transformation, Optical splitter, to send a signal down various fiber ways., Circulator, to tie in different parts, for example, an OADM and Optical intensifier. There is some types of service in optical system such as Circuitswitched. In optical fiber telecommunications, the ability to drop and add a single wavelength channel without having to convert all the channels in and out of electronics has been very useful; reconfigurable optical 
add-drop multiplexers (ROADMs) have allowed convenient expansion of systems, adding channels and reconfiguring networks as needed (Miller, 2013). An ensured data transfer capacity is allocated to every connection being accessible all the time the connection keeps going -Packet- switched. Data streams are separated into small packets of data. Packets are multiplexed together with parcels from other data streams inside the network and Packets are switched inside the network based on their destination. Two ways to increase the transmission capacity on a fiber (Baharudin et al., 2013):

- Increase the bit rate with time division multiplexing (TDM): many lower speed data streams are multiplexed into a higher-speed stream.

- Wavelength division multiplexing (WDM): transmit data simultaneously at multiple wavelengths TDM and WDM combined: tens of Tbsp.

Figure 1; shows the overview of optical networking evolution. The basic mechanism of communication in wavelength routed network is in the form of logical connection implemented using light path. Light path is viewed as a point-to-point all-optical communication channel from source to its destination, and it may span multiple fiber links. When optical networks operate as WRON, the following constraints are required (Mustafa, 2017):

- -Wavelength Continuity constraint: This constraint requires that the same wavelength must be allocated on all links on the chosen route from source to destination.

- -Distinct wavelength constraint: Connection sharing a common fiber must use distinct wavelength, which means that if two light paths share the same optical link they cannot have the same wavelength.

\section{SOFTWARE-DEFINED NETWORKING (SDN)}

SDN technology is a way to deal with PC networking that permits network administrators to programmatically initialize, control, change, and manage network behavior dynamically through open interfaces and reflection of lower-level functionality.

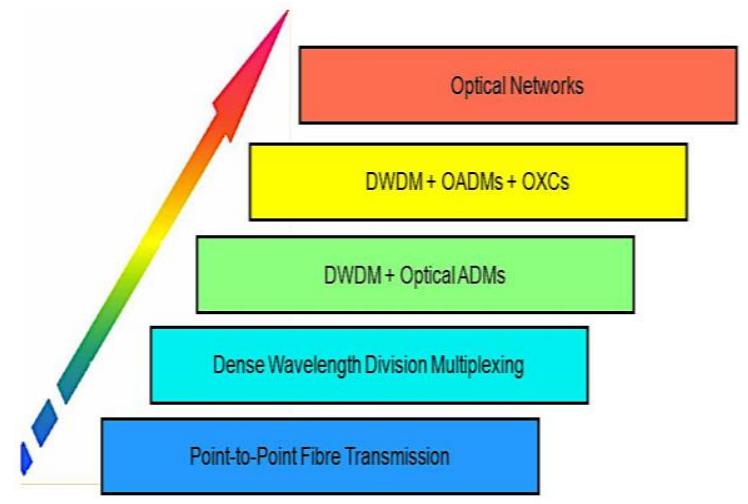

Fig. 1. Overview of optical networking evolution (Ramsey, 2015).

SDN is intended to address the way that the static design of traditional networks does not support the dynamic, adaptable processing and capacity needs of more current computing environments, for example, data centers. This is finished by decoupling or disassociating the framework that settles on choices about where activity is sent (the SDN controller, or control plane) from the basic frameworks that forward traffic to the destination (the data plane). SDN was generally connected with the OpenFlow protocol (for remote communication with network plane components with the purpose of deciding the way of network packets cross over network switches) since the last's rise in 2011. Since 2012, however, many organizations have moved far from OpenFlow, and have embraced different methods. These incorporate Cisco Systems' Open Network Environment and Nicira's network virtualization stage. SD-WAN applies comparable innovation to a wide range arrange (WAN). The origins of software- defined networking started not long after Sun Microsystems discharged Java in 1995 (Ramsey, 2015, Mustafa, 2017, Kantor et al., 2019). 
The Open Networking Foundation was established in 2011 to advance SDN and OpenFlow. At the 2014 Interop and Tech Field Day, software-defined networking was exhibited. Avaya used briefest way bridging and OpenStack as a robotized ground, expanding automation from the server farm to the end gadget, expelling manual provisioning from benefit delivery (Miller, 2013, Baharudin et al, 2013). By 2016, some in the industry thought that SDN had become a meaningless marketing term (Routray et al., 2016). SDN can be used for Virtualization, Orchestration, Programmable, Dynamic Scaling, Automation (Troubleshooting, Reduce downtime, Policy enforcement, Provisioning/Re- provisioning/Segmentation of resources, Add new workloads, sites, devices, and resources), Visibility (Monitor resources, connectivity), Performance (Traffic engineering/Bandwidth management, Capacity optimization, Load balancing, High utilization, Fast failure handling), Multi-tenancy and Service Integration.

The SDN state as an architecture indicating to be dynamic, reasonable, cost-effective, and versatile, looking to be appropriate for the high-data transmission, dynamic nature of the present applications. SDN structures decouple network control and sending capacities, enabling network control to become directly programmable and the fundamental framework to be abstracted from applications and network services.

\subsection{The SDN Architecture:}

The architecture of SDN is presented in Figure 2. It consists of three separate planes (Kantor et al., 2019):

i. Application Plane: Application plane is formed by programs that communicate requirements and network desired behavior to the SDN controller. These applications can build an abstracted view of the network by collecting information from the controller for decision making. These applications include: network analytics, network management, security and other business-related purposes.

ii. Control Plane: This plane is realized by the SDN controller. The controller is a logical entity that receives instructions from the Application Plane and controls the devices in the network to obtain given results. The controller also extracts information about the network from the devices and communicates back to the Applications Plane with an abstract view of the network and current traffic statistics.

iii. Data Plane: This plane forwards all the data within the network. It comprises SDN switches which consult the controller with every decision, a normal router takes independently. Particularly, when a new flow arrives, a switch asks the controller for the action to be performed on the flow, and then stores this information inside its flow table for further packets of the flow. Also, the architecture of SDN has a specific properties as follows:

- Directly programmable: Network control is directly programmable because it is decoupled from sending capacities.

- Agile: Abstracting control from sending lets administrators dynamically adjust networkwide traffic flow to meet changing needs.

- Centrally managed: Network intelligence is (logically) centralized in software-based SDN controllers that maintain a global view of the network, which appears to applications and policy engines as a single, logical switch.

- Programmatically configured: SDN lets network managers configure, manage, secure, and optimize network resources very quickly via dynamic, automated SDN programs, which they can write themselves because the programs do not depend on proprietary software. 
- Open standards-based and vendor-neutral: When implemented through open standards, SDN simplifies network design and operation because instructions are provided by SDN controllers instead of multiple, vendor-specific devices and protocols.

Figure 2; shows the architecture of SDN, while Figure 3; shows contains a graphical representation of the SDN architecture illustrating the application, control and data layers and interfaces.

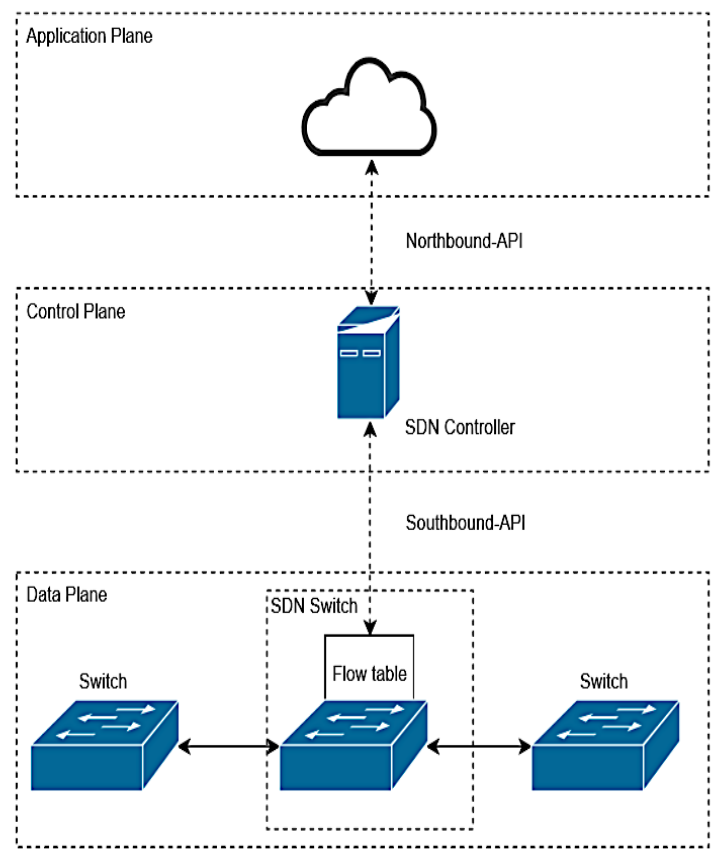

Fig. 2. The architecture of SDN (Kantor et al., 2019).

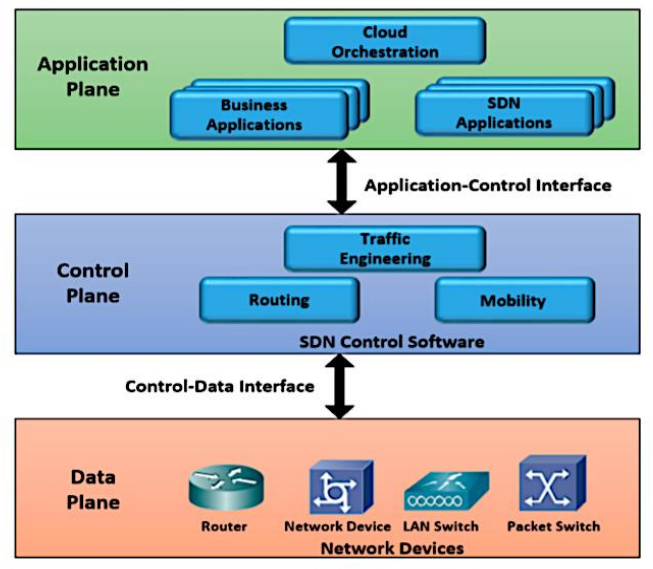

Fig. 3. SDN architecture illustrating the application, the layers and the interfaces (Sung, et al., 2016).

\section{EXPERIMENTAL STUDIES ON SDN APPLIED TO OPTICAL NETWORKS}

In various utilize networks; there frequently emerge certain activity streams, which make exceptional utilization of network transfer speed, in some cases to the point of starving other movement streams (traffic). These are regularly called elephant streams (flows) because of their sizable nature. The streams are described by being of relatively long length yet having a discrete start and end. The qualities can make it conceivable to foresee or plan these streams. Once detected, the objective is to reroute that traffic onto some type of equipment, such as, an all-optical network, which is provisioned particularly for expansive information offloads, for instance, this. OTNs are customized for these colossal volumes of packets traveling from one endpoint to another. Packet switches' capacity to route such elephant streams at packet level granularity is of no advantage, yet the weight an elephant stream puts on the packet switching network's links is intense. SDN has been effectively presented in the significant exertion has been as of late committed to stretch out the SDN architecture to efficiently operate on optical networks. Many researchers studied and used technique of SDN in Optical Network; Routray et al. (2016) presented the significance of SDN for network control and management. Optical networks have grown up in all spaces and its control and administration through SDN is very adaptable. SDN has been proposed for the control and administration of systems. SDN architecture can be thought to be comprised of three independent planes as showed in Figure 4. SDN gives a few points of interest to the control, operation, and administration of vast networks. It gives adaptability (flexibility) and readiness at each level of the network, and they show the SDN based hierarchy for optical networks in Figure 5. They have examined the 
advantages and execution issues of SDN for optical systems. SDN enhances the asset use in optical systems. With the expansion in the efficiency of the framework, the operational use is decreased. In general, SDN is required for entire resource optimization in optical networks (Routray et al., 2016).

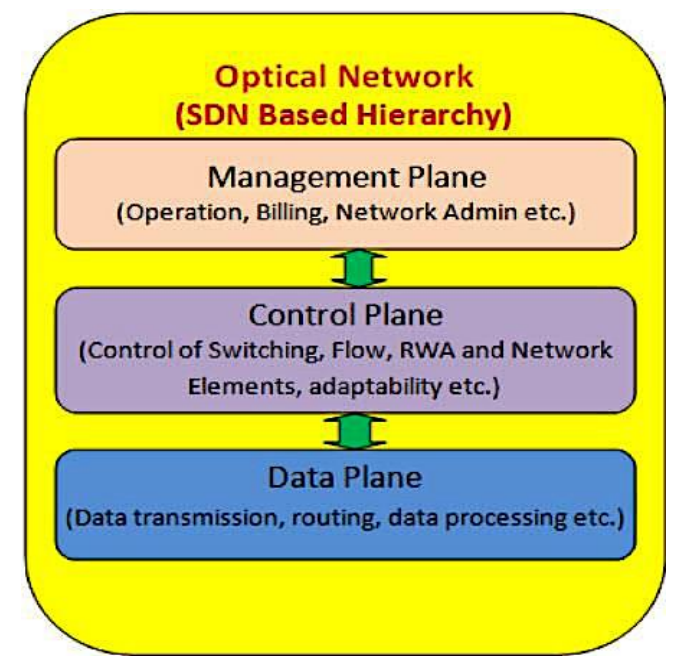

Fig. 4. General SDN Framework for communication networks (Routray et al., 2016).

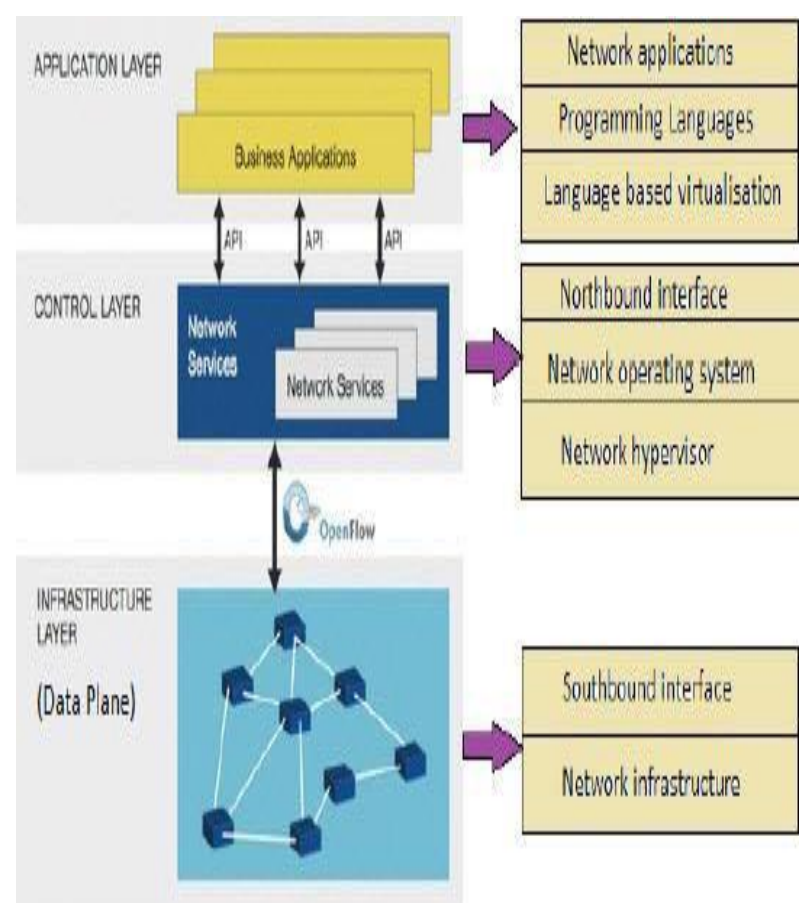

Fig. 5. SDN based hierarchy for optical networks (Routray et al., 2016).

Kantor et al. (2019) presented, compared, and contrasted solutions that utilize SDN in multi-layer network architectures. The main objective was to analyze multi- layer network architectures and show how these solutions coupled with SDN contribute to making future networks simple, flexible, and cost-effective. This survey fills the gap between the SDN concept and its possible implementations. There are a plethora of applications for a multi-layer SDN architecture. Although their adaptation and implementation may be difficult, the future of networking is bound with being softwareoriented. The key point of this survey is to present the newest proposals for multi-layer SDNs. However, not only such solutions have been presented, but also analyze their impact on network stability and complexity. The aspects of resource allocation, security, and resilience and network element provisioning are key elements when planning the network architecture. Moreover, the analysis of controllers, orchestrators, emulators, and testbeds provides additional information which is crucial for network optimization. The presented aspects were also analyzed for both, inter and intra-DC SDNs (Kantor et al., 2019).

Magalhães et al. (2017) proposed software defined networking (SDN) decouples the network control and forwarding functions enabling the network control to end up plainly straightforwardly programmable and the hidden foundation to be abstracted for applications and network administrations. In addition, elastic optical networking $(\mathrm{EON})$ technologies enable effective range use by apportioning variable transmission capacity to every client as indicated by their real needs. Specifically, adaptable transponders and reconfigurable optical add/drop multiplexers (ROADMs) are key components since they can offer degrees of opportunity to self-adjust as needs be. In this way, it is essential to configuration control strategies so as to optimize the equipment usage and offer high reconfigurability, adaptability and flexibility. They proposed and analyzed, utilizing a reproduction system, a strategy for limit expansion 
through optical power profile control for bury datacenter links that utilization existing metropolitan optical networks by misusing the global network view managed by SDN. The proposed technique embraces the weakening per channel to their base required all together have a higher OSNR fitting the best balance ensuring error free operation at the endpoint. The SDN control is utilized inside a simulation framework to dynamically adjust the power leveling procedure and modulation format to significantly expand the connection limit through OSNR optimization. Results demonstrated that it is possible to expand the network limit by $112 \%$ with a remaining tilt of $10.6 \mathrm{db}$. Therefore, the strategy has been appeared to improve the limit of every single optical connection through a metropolitan area network, which will be valuable for between datacenter links. Moreover, enabling dynamic adaptive capacity maximization adds strength and intelligence to WDM optical networks without the requirement for exchange any device (Magalhães et al., 2017).

Amazonas et al. (2016) exploited the software defined elastic optical networks (SD-EONs) aiming at identifying its state of art and the future challenges in terms of efficient dynamic resources assignment. In addition, they presented some up-to-date information concerning the perspectives that operators carry out real deployments of this technology in the core network where currently predominate GMPLS solutions. They analyzes the current scenario and the (imminent) future challenges that operators are facing have proposed what could be a comprehensive extended SDN architecture. They showed that there are plenty of research studies addressing the problem of finding SDN based Elastic Optical Network solutions. Most of these studies also show that SDN schemes perform better (e.g. reduced light- path setup time) than GMPLS-based schemes. Nevertheless, for what refers to the deployment of a SDN based Elastic Optical Network they have mainly to face two challenges, namely: 1- GMPLS is completely un-usable as a Unified Control Plane, but at the same time there is a great resistance of the network administrator to remove it, especially from the core network, where almost all the operators have installed it. This fact, reduces the possibilities that in the short time, SDN- EON to be deployed in the core of the public network infrastructure. 2- There is no a clear evidence that a control plane based on the SDN framework fully relying on a centralized controller that, besides path computation and spectrum defragmentation, is also in charge of light path establishment and management is able to provide benefits with respect to GMPLS/PCE (Amazonas et al., 2016).

Blendin et al. (2016) approached to superimposing SDN to increase the efficiency of the packet layer in elastic optical carrier networks was introduced. Its ability to keep the resilience and reliability characteristics of proven MPLS technology is investigated while enabling centralized SDN control for failure recovery. If optical links offer reduced capacity after link failure, the SDN controller selectively overrides the MPLS infrastructure to utilize of the remaining link capacities more efficiently. They proposed to enable source routing by introducing Segment Routing to existing MPLS routers and superimposing SDN functionality at the edge of the MPLS network (Segment Routing-based approach with superimposed Softwaredefined Networking (SDN) to allow the IP network to benefit from new features. The logically centralized SDN control plane is used for interfacing with the controller of the optical network to receive information about link bandwidth changes. The contributions of their studied are: 1- A description of requirements for 
IP-optical cooperation when repairing link failures with subsequent capacity reductions in EONs. 2- A minimally invasive, gradually deployable, superimposing SDN-based approach that complements MPLS for steering traffic off a network link during capacity reductions. 3- An investigation into gradual deployment strategies to determine the smallest number of changes required when introducing the system to existing networks. The packet layer has to be involved to exploit the potential of bit-rate flexible optical transceivers in elastic optical networks. The results show that gradually deploying new technologies to PoPs is a promising approach in the investigated network. Finally, superimposing SDN over an existing network is a viable approach to add new features to a network while minimizing its impact on the reliability characteristics of the network (Blendin et al., 2016).

Routray et al. (2016) presented the importance of SDN for network control and management. Optical networks have grown up in all domains and its control and management through SDN are quite flexible. The benefits and implementation issues of SDN for optical networks have been analyzed. SDN optimizes resource utilization in optical networks. With the increase in the efficiency of the system, the operational expenditure is reduced. Overall, SDN is required for entire resource optimization in optical networks. SDN is a network architecture that was developed to eliminate the existing configuration complexity of the control and transport layer network protocols. By reducing the configuration complexity SDN aims to enable the network engineers and administrators to respond quickly to changing business requirements. To achieve this, SDN introduces the concept of centralized control in networking (Routray et al., 2016).

Sadasivarao et al. (2016) presented their observations deploying SDN-enabled solutions in operational Tier-1 carrier networks. This includes technical and operational issues that are of prime importance to service providers. Their goals is to provide perspectives deploying SDN applications in operational optical transport networks, primarily in the WAN context. They presented their experiences deploying OTSv in Tier-1 carrier networks, along with two key use cases for which, SDN techniques would be a good fit: Layer 1 Virtual Private Networks and Port/flow services. In addition, they viewed on other critical aspects such as the role of embedded control plane in an SDN environment and integration/development models used by carriers (DevOps v/s NetOps). Specifically; they focused on two archetypal customer use cases that they had observed from the point of view of optical transport applications. In addition, other key aspects such as the role of embedded control plane in SDN are discussed. Development models used by service providers and how this plays a crucial/symbiotic role between the vendor (enhancing platform data \& control plane features) and the service provider (rolling out SDNenabled services) are considered. Concluded show that synopsis on the state-of-affairs, and the role of standardization in the SDN ecosystem. They expected traditional embedded control plane to be in use while carriers devise strategies for a seamless migration to SDNbased network operations, management and control (Sadasivarao et al., 2016).

Santos (2016) presented a novel architecture for SDN controllers utilized in the optical domain. The proposition suggests part the full network administration over a league of sub- network controllers, all helpfully planned by a progressively superior controller. In the specific situation, the network controller, whose primary duty lies in the proficient configuration of the network hardware, is a critical component to the SDN architecture. Normally, 
such controllers are centralized components that detail the total information of the network. However, for substantial measured networks, such focus can prompt lengthier operations, heavier flexibility plans, and accessibility of extreme storage and processing capacity. To hinder these issues and promote scalability, they presented another controller architecture based on a federation of multiple subnetwork, each overseeing just an area of the network, helpfully planned by a hierarchically superior controller. A clustering algorithm, based on single-linkage, was produced to make sub-networks under various gathering approaches. In order to survey the effect of utilizing the combined approach in an optical network, they utilized a reference topology to determine the amount of routed requests and regeneration cards required. The results obtained showed that the federated architecture performance only gets closer to the traditional single-controller scheme when a reasonable number of end-to-end paths are computed. Moreover, the usage of increased sub- network cardinalities are shown beneficial to reduce blocking and regeneration. Conversely, increased traffic loads tend to impair the routing efficiency (Santos, 2016).

De Siqueira et al. (2015) introduced a novel approach based mechanism to provide context- aware network wide strategies to Software Defined Networking (SDN) applications, executed with policy flow based in view of property graph models. The proposition has been approved in a transport SDN controller, supporting optical network virtualization by means of cutting of physical resources, such as, nodes, connections and wavelengths, through utilize case tried demonstrations of policy implementation for SDN applications, including optical adjustment and virtual optical network control. Therefore, they exhibited two use instances of strategy requirement for SDN applications, in particular, Optical Equalization. SDN Application (EQUAL-APP) and Virtual Optical Network Configuration and Restoration SDN Application (VONCR-APP). One of the reasons for this work is to characterize and exhibit requirement of policies to SDN applications. Subsequently, the following Transport SDN (T-SDN) applications have been considered as use cases for approval purposes. A simulation-assisted local policy mechanism has been proposed and illustrated, which permits network components to dynamically respond to specific situations if there should be an occurrence of disappointment in communications with the T-SDNC or if there should arise an occurrence of strict timing requirements (De Siqueira et al., 2015).

Ma et al. (2015) studied the survivability of virtual optical network embedding in an alternate area (domain) based on Software Defined Network. Optical network virtualization in various regions can advance the productivity of physical foundation among various clients and applications. An integrated control model is set up in this investigation based on the Network Operating System architecture, which isolates the route analysis and data transmission. They additionally proposed a disappointment probability Memetic algorithm to deal with the network embedding issue by comparing with the existing baseline algorithm. The examination proposes an incorporated design in view of the hierarchical software defined network (SDN) control plane. The significant point in this study the contribution is considering about every one of the constraints of opaque VONE and advancing an enhanced model for the VON in view of SDN control architecture. They also develops a novel algorithm called failure probability memetic algorithm (FP-MA) to map VONs on the physical substrate network connect with little failure probability (FP) and views 
the limiting FP as objective. They solved the issue of virtual optical system inserting in multi- domain networks by receiving a NOX control plane based on SDN, and they additionally propose an effective memetic calculation with the goal of limiting the aggregate network failure probability. Simulation results demonstrate that the enhanced model has a superior execution in multi-domain issues and the memetic algorithm accomplishes lower average VONFP than other existing algorithms (Ma et al., 2015).

Paolucci et al. (2015) presented a novel hierarchical OAM architecture is proposed, that enables multi-level OAM entities to provide OAM Handler with effective information, obtained by filtering several OAM messages at each layer, so that the overload of OAM Handler is avoided. Moreover, the NETCONF protocol, typically used for SDN-based node configuration purposes, is proposed and utilized as OAM protocol, in order to achieve high degree of convergence and limit the number of utilized protocols. Scalability is assured by enabling multi-level correlation and aggregation of OAM data towards the extended OAM Handler, which is able to identify QoT degradation sources and the related affected resources and, therefore, trigger the SDN control plane to react with the most appropriate countermeasures. Proposed OAM architecture is implemented and experimentally evaluated in a QoT degradation use case, showed that multi-level localization and local correlation of events allow aggregated, fast and scalable OAM information set provided to OAM Handler, where OAM infrastructure successfully localizes and derives degradation forecast before traffic data loss occurs through a single aggregated NETCONF message (Paolucci et al., 2015).

Finally, and although mainly confined to scenarios where the sub-network count is low, the clustering policy can negatively influence the results. The summary of all previous literature review is presented in Table 1.

\section{DISCUSSION}

Finally, this survey fills the gap between the SDN concept and its possible implementations. The summary of all previous literature review is presented in Table 1, and from these information, the problems zone was regarding the analyzing network architectures and show how these solutions coupled with SDN contribute to Table 1. (Overview of Optical Network using SDN)

\begin{tabular}{|c|c|c|c|c|c|}
\hline 点 & $\frac{5}{0}$ & 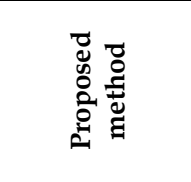 & 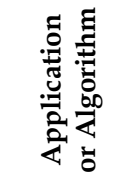 & 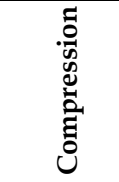 & 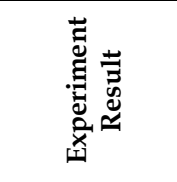 \\
\hline $\begin{array}{l}\text { Magal } \\
\text { hães } \\
\text { et al. } \\
\text { (2017) }\end{array}$ & $\begin{array}{l}\text { Analyzing } \\
\text { multi- } \\
\text { layer } \\
\text { network } \\
\text { architectu } \\
\text { res and } \\
\text { show how } \\
\text { these } \\
\text { solutions } \\
\text { coupled } \\
\text { with SDN } \\
\text { contribute } \\
\text { to making } \\
\text { future } \\
\text { networks } \\
\text { simple, } \\
\text { flexible, } \\
\text { and cost- } \\
\text { effective. }\end{array}$ & $\begin{array}{l}\text { SDN } \\
\text { decouples the } \\
\text { network } \\
\text { control and } \\
\text { forwarding } \\
\text { functions } \\
\text { enabling the } \\
\text { network } \\
\text { control to end } \\
\text { up plainly } \\
\text { straightforwar } \\
\text { dly } \\
\text { programmabl } \\
\text { e and the } \\
\text { hidden } \\
\text { foundation to } \\
\text { be abstracted } \\
\text { for } \\
\text { applications } \\
\text { and network } \\
\text { administratio } \\
\text { ns }\end{array}$ & $\begin{array}{l}\text { The } \\
\text { multi- } \\
\text { layer } \\
\text { SDN } \\
\text { inter and } \\
\text { intra-DC } \\
\text { SDNs. }\end{array}$ & $\begin{array}{l}\text { OSNR } \\
\text { threshol } \\
\text { d vector }\end{array}$ & $\begin{array}{l}\text { The analysis } \\
\text { of } \\
\text { controllers, } \\
\text { orchestrator } \\
\text { s, emulators, } \\
\text { and testbeds } \\
\text { provides } \\
\text { additional } \\
\text { information } \\
\text { which is } \\
\text { crucial for } \\
\text { network } \\
\text { optimization } \\
\text {. The } \\
\text { presented } \\
\text { aspects were } \\
\text { also } \\
\text { analyzed for } \\
\text { both, inter } \\
\text { and intra- } \\
\text { DC SDNs. } \\
\text { manipulatin } \\
\text { g the loss } \\
\text { profiles of } \\
\text { the } \\
\text { ROADMs in } \\
\text { the network } \\
\text { can } \\
\text { yield optical } \\
\text { signal-to- } \\
\text { noise ratio } \\
\text { (OSNR) } \\
\text { improvemen } \\
\text { ts up to } 10 \\
\text { dB leading } \\
\text { to an } \\
\text { capacity. }\end{array}$ \\
\hline
\end{tabular}




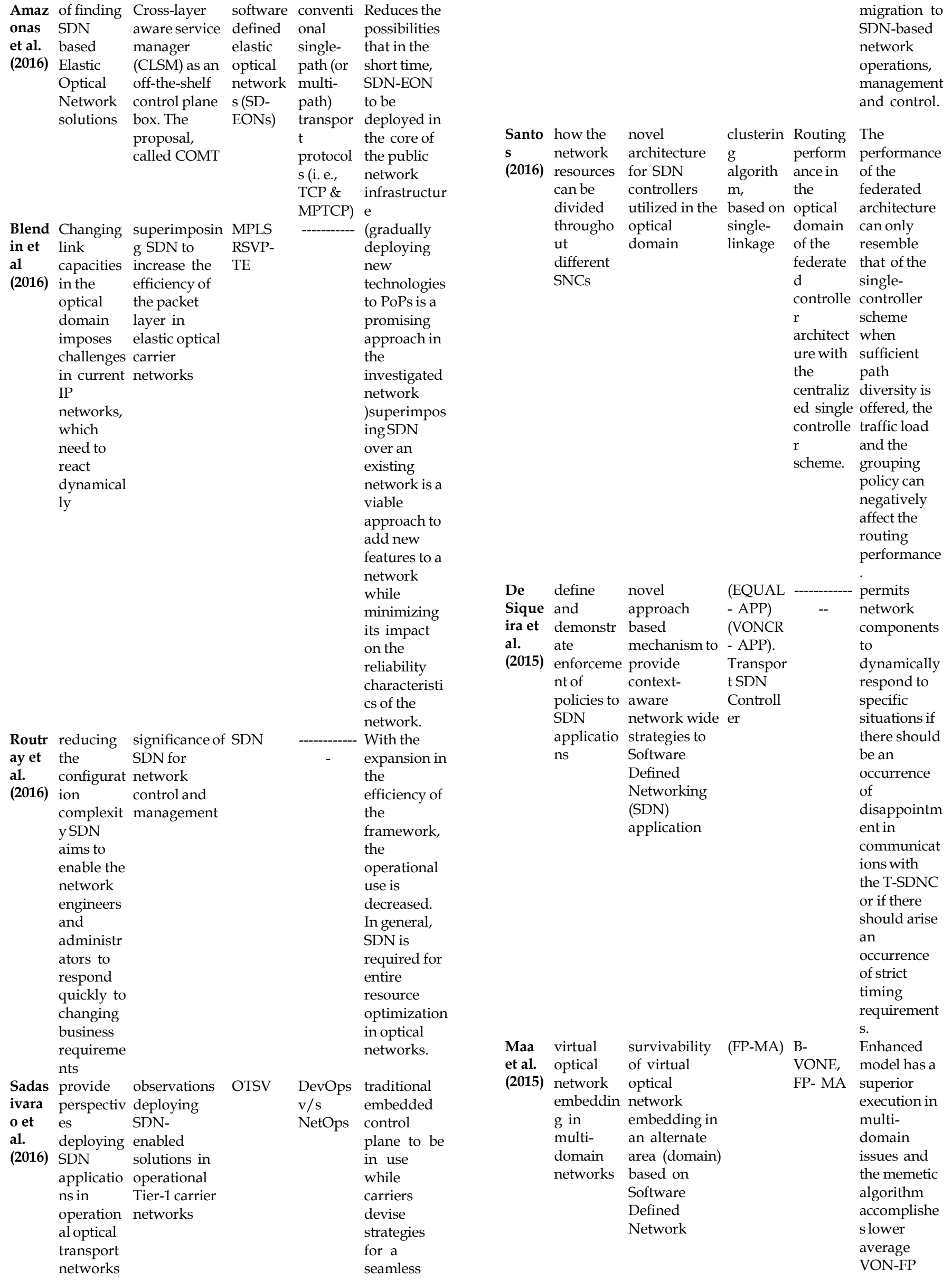


than other existing algorithms.

\begin{tabular}{|c|c|c|c|c|}
\hline $\begin{array}{l}\text { Paolu } \\
\text { cci et } \\
\text { al. } \\
(2015)\end{array}$ & $\begin{array}{l}\text { guarantee novel } \\
\text { quality of hierarchical } \\
\text { transmissi OAM } \\
\text { on(QoT), architecture } \\
\text { novel } \\
\text { Operation } \\
\text { Administr } \\
\text { ation and } \\
\text { Maintena } \\
\text { nce } \\
\text { (OAM) } \\
\text { solutions } \\
\text { are } \\
\text { necessary } \\
\text { with } \\
\text { respect to } \\
\text { existing } \\
\text { standard } \\
\text { managem } \\
\text { ent } \\
\text { protocols }\end{array}$ & $\begin{array}{l}\text { OAM } \\
\text { infrastru } \\
\text { cture } \\
\text { design } \\
\text { based on } \\
\text { NETCO } \\
\text { NF }\end{array}$ & ----- & $\begin{array}{l}\text { OAM } \\
\text { infrastructur } \\
\text { e } \\
\text { successfully } \\
\text { localizes } \\
\text { and derives } \\
\text { degradation } \\
\text { forecast } \\
\text { before traffic } \\
\text { data loss } \\
\text { occurs } \\
\text { through a } \\
\text { single } \\
\text { aggregated } \\
\text { NETCONF } \\
\text { message }\end{array}$ \\
\hline
\end{tabular}

making future networks simple, flexible, and costeffective, and with the focusing on reducing the configuration complexity SDN and improves optical link capacity utilization in the network that is suitable for enhancing inter data-center networking. While the proposed methods for solving the issues were about the analyze the suggested methods and their impact on network stability and complexity to increase the efficiency of the packet layer in elastic optical carrier networks. Also, the proposed methods mentioned the significance of SDN for network control and management, observations deploying SDN- enabled solutions. By other words and to cover another side the proposed methods were used to study the survivability of virtual optical network embedding in an alternate area (domain) based on SDN, and the analysis of controllers, orchestrators, emulators, and testbeds provides additional information which is crucial for network optimization.

\section{CONCLUSION}

The concept of SDN can be used to efficiently manage and control the whole network in all its layers. As the surveyed solutions show, applying SDN to multiple layers provides new possibilities. In this survey, the solutions that utilize SDN in network architectures have been presented, compared, and contrasted. The main goal was to describe the significant developments in the region of communication using $\mathrm{SDN}$ in the optical network, provide a deep and comprehensive perception of this model, its related technologies, and its areas of application, as well as significant issues that need to be solved towards providing its success. There are a plethora of applications for a multi-layer SDN architecture. Although their adaptation and implementation may be difficult, the future of networking is bound with being software-oriented.

The key point of this survey is to present the newest designs for multi-layer SDNs. The features of resource allocation, security, and resilience and network element provisioning are key elements when planning the network architecture. Moreover, the analysis of controllers, orchestrators, emulators, and testbeds provides additional information that is essential for network optimization. Finally, the issues, which will play a significant role in future multi-layer SDNs have been presented also.

\section{REFERENCES}

1. Kreutz, D., et al., (2015). Software-defined networking: A comprehensive survey. Proceedings of the IEEE, vol.103, no. 1, pp. 14-76, Jan. 2015, doi: 10.1109/JPROC.2014.2371999. Retrieved from https:/ / ieeexplore.ieee.org/document/6994333.

2. Mustafa, Firas M., and Al-Jumailly, Tariq A. , (2017). Buffer less All-Optical WDM Networks with Dynamic Traffic, Academic Journal of Nawroz University (AJNU), Vol.6(1), No.(10), Pages:13-25. DOI: $10.25007 /$ issn.2520-789X. Retrieved from https://journals.nawroz.edu.krd/index.php/ajnu/index

3. Kantor, Mirosław, et al., (2019). A survey on multi-layer IP and Optical Software-Defined Networks, Computer Networks, doi: 10.1016/j.comnet.2019.06.022. $\quad$ Retrieved from https://www.sciencedirect.com/science/article/abs/pii/S138 9128618314312?via\%3Dihub

4. Miller, David A. B., (2013). Reconfigurable add-drop multiplexer for 
Academic Journal of Nawroz University (AJNU), Vol.9, No.2, 2020

spatial modes. Optics Express, 21(17) ,20220. doi:10.1364/oe.21.020220. Retrieved from https:// www.osapublishing.org/oe/abstract.cfm?uri=oe-21$17-20220$

5. Baharudin, Nor N., et al., (2013). Review on multiplexing techniques in bandwidth utilization, Middle-East Journal of Scientific Research 18(10):1510-1516,2013 doi:10.5829/idosi.mejsr.2013.18.10.12422. Retrieved from https://www.idosi.org/mejsr/mejsr18(10)13/21.pdf

6. Ramsey, Nathaniel, (2015), Optical Networking. Applied Optoelectonics Center, School of Electronic and Communications Eng., Dublin Institute od Technology. Retrieved from https://slideplayer.com/slide/4634737/

7. Routray, Sudhir K., et al. (2016). Software defined networking for optical networks. In Distributed Computing, VLSI, Electrical Circuits and Robotics (DISCOVER), IEEE. DOI: 10.1109/DISCOVER.2016.7806260. . Retrieved from https://ieeexplore.ieee.org/document/7806260

8. Sung, Yunsick, et al., (2016). A Taxonomic Modeling of Security Threats in SDN for Future Sustainable Computing. MDPI journals, Sustainability, Volume 8, Issue 9 (September 2016). doi: 10.3390/su8090919. Retrieved from https://www.mdpi.com/2071-1050/8/9/919/htm

9. Magalhães, Eduardo, et al., (2017). SDN control of optical nodes in metro networks for high capacity inter-datacenter links. Optics Communications, p: 173-180. doi: 10.1016/j.optcom.2017.06.026. Retrieved from https://doi.org/10.1016/j.optcom.2017.06.026

10. Amazonas, José Roberto de Almeida, et al. (2016). Technical challenges and deployment perspectives of SDN based elastic optical networks. In Transparent Optical Networks (ICTON), $18^{\text {th }}$ International Conference IEEE. Doi:10.1109/ICTON.2016.7550418. Retrieved from https://ieeexplore.ieee.org/document/7550418

11. Blendin, Jeremias, et al. (2016). Enabling efficient multi-layer repair in elastic optical networks by gradually superimposing SDN. In Network and Service Management (CNSM), 12 ${ }^{\text {th }}$ International Conference. IEEE. DOI:10.1109/CNSM.2016.7818407. Retrieved from

https://ieeexplore.ieee.org/document/7818407?section=abstra $\underline{\mathrm{ct}}$

12. Sadasivarao, Abhinava, et al., (2016). Demystifying SDN for Optical Transport Networks: Real-World Deployments and Insights. In Global Communications Conference (GLOBECOM), IEEE. DOI: 10.1109/GLOCOM.2016.7841727. Retrieved from https://ieeexplore.ieee.org/document/7841727

13. Santos, João, (2016). Scalable design of SDN controllers for optical networks using federation-based architectures. In
Networks and Optical Communications (NOC), 21 ${ }^{\text {st }}$ European Conference. IEEE. DOI: 10.1109/NOC.2016.7506988. Retrieved from https://ieeexplore.ieee.org/document/7506988

14. De Siqueira, Marcos Antonio, et al., (2015). Providing optical network as a service with policy-based transport SDN. Journal of Network and Systems Management, 23(2): p. 360-373. Retrieved from https://link.springer.com/article/10.1007/s10922-0149320-x

15. Ma, Jiachen., et al. (2015). Virtual optical network embedding with SDN architecture based on memetic algorithm. In Computer Science and Network Technology (ICCSNT), $4^{\text {th }}$ International Conference on IEEE. DOI:10.1109/ICCSNT.2015.7490917. Retrieved from https://ieeexplore.ieee.org/document/7490917

16. Paolucci, Francesco, et al. (2015). Hierarchical OAM infrastructure for proactive control of SDN-based elastic optical networks. In Global Communications Conference (GLOBECOM), IEEE. DOI: 10.1109/GLOCOM.2015.7417223. Retrieved from https://ieeexplore.ieee.org/document/7417223 